\title{
El aula invertida como recurso didáctico en el contexto costarricense: estudio de caso sobre su implementación en una institución educativa de secundaria
}

Araya-Moya, Suhelen María; Rodríguez Gutiérrez, Ana Lucía; Badilla Cárdenas, Nancy Fabiola; Marchena Moreno, Katherine Cristina

El aula invertida como recurso didáctico en el contexto costarricense: estudio de caso sobre su implementación en una institución educativa de secundaria

Revista Educación, vol. 46, núm. 1, 2022

Universidad de Costa Rica, Costa Rica

Disponible en: https://www.redalyc.org/articulo.oa?id=44068165004

DOI: https://doi.org/10.15517/revedu.v46i1.44333

\section{(ㄷ) $\odot \Theta \Theta$}

Esta obra está bajo una Licencia Creative Commons Atribución-NoComercial-SinDerivar 3.0 Internacional. 


\title{
El aula invertida como recurso didáctico en el contexto costarricense: estudio de caso sobre su implementación en una institución educativa de secundaria
}

\author{
Implementing Flipped Classrooms as an Educational Resource in Costa Rica: A High School Case Study
}

Subelen María Araya-Moya

Universidad de Costa Rica, Costa Rica

suhelen.araya@ucr.ac.cr

DOI: https://doi.org/10.15517/revedu.v46i1.44333

Redalyc: https://www.redalyc.org/articulo.oa? id $=44068165004$

iD https://orcid.org/0000-0002-5333-3349

Ana Lucía Rodriguez Gutiérrez

Universidad de Costa Rica, Costa Rica

ana.rodriguezgutierrez@ucr.ac.cr

iD https://orcid.org/0000-0002-3636-9577

Nancy Fabiola Badilla Cárdenas

Universidad de Costa Rica, Costa Rica

nancy.badilla@ucr.ac.cr

iD https://orcid.org/0000-0002-9360-9071

Katherine Cristina Marchena Moreno

Universidad de Costa Rica, Costa Rica

katherine.marchenamoreno@ucr.ac.cr

iD https://orcid.org/0000-0002-6529-4148

Recepción: 14 Noviembre 2020

Aprobación: 02 Febrero 2021

\section{Resumen:}

El aula invertida es un modelo pedagógico alternativo que busca optimizar el tiempo invertido en las clases presenciales, devolver el papel activo a las personas estudiantes y generar espacios de enseñanza-aprendizaje más significativos. La presente investigación exploró la implementación del aula invertida en la institución educativa costarricense CEDES Don Bosco; para ello se seleccionó a tres profesionales de esta institución que han sido pioneros y pioneras en la aplicación de dicha metodología. Se indagó, a través de entrevistas semiestructuradas, sus experiencias y trayectoria en el tema; posteriormente, se llevó a cabo la codificación de la información obtenida mediante la identificación primaria de subcategorías temáticas, las cuales dieron paso al surgimiento a las 7 categorías principales (conceptualización del aula invertida, implementación, aplicación, beneficios y aportes, rol del docente, actitudes del estudiantado y retos) analizadas en esta investigación. Se encontró que la aplicación del aula invertida en la institución ha implicado un proceso de capacitación, adaptación y acompañamiento, que se traduce en retos que se han resuelto sobre la marcha, y beneficios que disfrutan tanto las personas entrevistadas como la comunidad estudiantil participante. Asimismo, se halló que el estudiantado involucrado, inicialmente, presentó actitudes de indiferencia o poca aceptación, promovidas por una cultura estudiantil facilista; sin embargo, conforme se adaptaron al modelo, desarrollaron mayor compromiso y responsabilidad para realizar las tareas previas a la clase presencial. Como parte de las recomendaciones se destaca la necesidad del compromiso por parte del personal docente, su anuencia a la investigación, capacitación y planificación; así como, su profesionalismo empático y flexible, que se adapta a las estrategias y herramientas utilizadas al contexto y particularidades del alumnado. Además, se hace evidente la importancia de que las personas profesionales en educación se mantengan actualizadas, de forma particular en el uso de las nuevas tecnologías de información y comunicación, así como en el uso de modelos pedagógicos alternativos. Por último, se concluye que este modelo, a pesar de involucrar tecnología y representar retos, es adaptable a cualquier contexto educativo.

Palabras ClaVE: Educación, Innovación pedagógica, Metodologías activas, Aula invertida, Recurso didáctico.

\section{Abstract:}


Flipped classrooms are alternative educational models aimed at making the best use of face-to-face classroom time and returning to a model where students play an active role. The objective is to create more meaningful teaching and learning opportunities. This study analyzed flipped classrooms at CEDES Don Bosco High School in Costa Rica. Three teachers from that institution, considered to be pioneers and experienced in this type of methodology, were selected and their responses from semi-structured interviews analyzed. Data was collected based on topic subcategories that were stratified into the following seven classifications which were analyzed as part of this study: the concept of flipped classrooms, implementation, application, benefits and contributions, role of teachers, attitudes of students and challenges faced. The use of flipped classrooms at this institution has required training, adaptation and support to face the many challenges resolved along the way. It has also entailed benefits for the interviewees the participating student body. Although students were hesitant and indifferent, at first, a result of having been exposed to a previous mediocre learning culture, as they adapted to the model, they developed greater commitment and responsibility in performing the assigned tasks prior to face-to-face classes. Recommendations include greater a commitment by the teaching staff who should make an effort for further research, training and planning of activities. Teachers must also be more empathetic, flexible and willing to adapt to the particular strategies and tools that should be used for each student context. It is also essential for teachers to remain abreast of new information and communication technology and the use of alternative pedagogical models. Although this model requires technology and also faces challenges, it is readily adaptable to any educational context.

KEYWORDS: Education, Pedagogical Innovation, Active Methodologies, Flipped Classrooms, Educational Resource.

\section{INTRODUCCIón}

A través del contexto provocado por la pandemia del COVID-19 en Costa Rica, se ha evidenciado la necesidad de la virtualización de contenidos en el sistema educativo y de fomentar una cultura de responsabilidad y participación activa en la adquisición de conocimientos por parte del estudiantado, roles que pueden ser indispensables ante eventos de crisis. Como se menciona en la 6ta edición del Estado de la Educación Costarricense (2017), desde hace algún tiempo en el país se busca una amplitud en la promoción de nuevas propuestas curriculares que promuevan en el estudiantado una serie de "habilidades para la vida, así como, el manejo de herramientas que les permitan integrarse plenamente como ciudadanos del mundo actual." (p. 52). Entre las habilidades más importantes que destaca este informe se encuentran "las habilidades comunicativas, la indagación, el pensamiento crítico, la innovación, la apropiación de las TICs, el trabajo colaborativo y la responsabilidad social", de las cuales las últimas tres son elementos que se logran desarrollar cuando se implementan metodologías activas, como el aula invertida.

Aunado a lo anterior, resulta de gran relevancia la creciente demanda de formar futuras personas profesionales en educación, capaces de hacer transposiciones didácticas ajustables a las necesidades estudiantiles inmediatas, donde la importancia de la digitalización o el uso de diferentes recursos didácticos es innegable. Por su parte, González (2001) argumenta que, desde finales del siglo pasado, la sociedad ha atravesado una era digital que continúa en gran progreso. Ante esto, el autor plantea que el principal desafío de la sociedad, en materia de educación, se dirige a generar un cambio que vaya introduciendo en el modelo pedagógico de enseñanza y aprendizaje unas dosis crecientes de autoaprendizaje mediante el uso de la tecnología disponible.

Ante estas realidades, el modelo de aula invertida o fipped classroom se presenta como una opción viable en el proceso educativo regular, debido a su amplia documentación y aplicación internacional, pues busca invertir los roles y los momentos de la enseñanza tradicional. Como se citó en Coufal (2014), Lage et al. (2000) y Talbert (2012) citado por Martínez-Olvera et al., (2014), el aula invertida propone que la persona estudiante sea responsable de acceder a los conocimientos facilitados por las personas docentes a través de herramientas multimedia en un espacio extra-clase; de esta forma los espacios de clase presencial se orientan al uso de métodos interactivos de trabajo colaborativo, aprendizaje basado en problemas y realización de proyectos, con el fin de reforzar el proceso de aprendizaje (Martínez-Olvera et al., 2014).

De esta forma, la necesidad de proponer cambios resulta fundamental para prever un proceso educativo donde el estudiantado tenga una participación más activa en la adquisición de conocimientos y, ante lo cual, 
el aula invertida promueve que sea la propia persona quien busque la información para luego ser socializada en el aula por medio de un aprendizaje colaborativo, y a través de la guía del personal docente.

En Costa Rica se tiene conocimiento de que la institución Centro de Educación Salesiana (CEDES) Don Bosco, ubicada en Concepción Arriba de Alajuelita, ha promovido, desde hace algunos años atrás, la metodología de aula invertida con el equipo docente de la institución. Debido a la escasez de literatura sobre la aplicación de dicha metodología en el contexto educativo costarricense, es fundamental abordar ejemplos prácticos para conocer casos inmediatos a la realidad educativa, con miras al mejoramiento de su praxis y, eventualmente, su replicación en otras instituciones.

Resulta importante la implementación de estrategias innovadoras en el contexto actual de la era digital, y por las necesidades producidas a raíz de la pandemia. Este artículo pretende vislumbrar el aula invertida como una opción pedagógica innovadora, ya utilizada en una institución educativa nacional. Por tanto, el objetivo general del presente estudio se conduce a explorar la implementación del aula invertida en la institución educativa costarricense CEDES Don Bosco con respecto a su forma de aplicación y a las ventajas y retos experimentados de su puesta en práctica.

\section{ANTECEDENTES}

Respecto a la aplicación del modelo a nivel internacional, y de forma particular dentro de la educación superior, una variedad de estudios da cuenta de la diversidad de disciplinas que han utilizado esta metodología para los procesos de enseñanza y aprendizaje en distintas universidades. La literatura más reciente reúne la aplicación del modelo en asignaturas de álgebra y matemáticas (Barros y Martínez, 2018), cálculo diferencial e integral (Pavanelo y Lima, 2017), medicina (Domínguez et al., 2017), termodinámica térmica, enfermería ciencias psicosociales aplicables a la salud (Sabater et al., 2017), inglés y odontología (Moya y Williams, 2016).

Los principales resultados de estos estudios evidencian que el modelo hace uso de escenarios mucho más favorables para la adquisición de conocimientos en las diferentes asignaturas, lo que contribuye a fomentar una actitud estudiantil más activa y en la que se retoma un proceso de formación centrado en el estudiantado (Aguayo et al., 2019).

Además, el aula invertida incide positivamente en el rendimiento académico, la creatividad, la participación de clase (Fúneme, 2019), la motivación que presenta el estudiantado (Mendaña et al., 2017), y en el aprendizaje significativo y la satisfacción estudiantil (Peña et al., 2017) y docente.

Los principales retos encontrados giran en torno a la gran demanda de tiempo que implica para la persona docente preparar el proceso educativo y a las reacciones estudiantiles negativas iniciales sobre el modelo, debido a la experiencia con metodologías tradicionales, traducidas en falta de disposición, ansiedad ante el cambio e incertidumbre.

La literatura referente a la aplicación del modelo en entornos de educación secundaria refleja un amplio rango de aplicabilidad en diversidad de asignaturas con resultados efectivos, tales como matemáticas, idiomas, religión, ciencias naturales y materias varias de educación básica. En cuanto a los resultados obtenidos con el alumnado, se logró evidenciar la importancia que juega la persona docente como mediadora del proceso educativo, y la implicación que tiene el uso de metodologías activas y de la tecnología (Gonçalves y Quaresma, 2018). También, con el uso de esta metodología se logró apreciar un mayor protagonismo del estudiantado en su proceso de aprendizaje y una interacción más activa en el uso de las TICs (Galindo y Badilla, 2016).

Por otro lado, el estudio de Barao y Palau (2016) arrojó resultados en los que se muestra que la comunidad estudiantil, que se expuso al modelo de aula invertida, visualiza mejoras importantes en sus evaluaciones académicas, así como la experimentación de diversos aspectos positivos, tales como tener más tiempo en casa para estudiar, menor gasto de tiempo en la visualización del contenido educativo, aclaración de dudas, mayor trabajo en equipo de forma colaborativa y autónoma, y mejoramiento en la interacción docente-discente y en la actitud y comportamiento estudiantil. 
A pesar de lo anterior, se visualizaron una serie de limitaciones. La principal de ellas es el cambio que la persona docente debe configurar en su esquema de una enseñanza tradicional, ya que la innovación tecnológica requiere en muchos casos de ese cambio. En este contexto, también se plantea la necesidad de considerar que una gran parte del alumnado puede no tener acceso a internet o tecnologías (Barao y Palau, 2016). Otra de las limitaciones encontradas es la carente apropiación de las tecnologías activas que tiene el profesorado y otras personas involucradas en los procesos académicos (Gonçalves y Quaresma, 2018).

En el ámbito nacional, la poca investigación encontrada refiere la aplicación del aula invertida principalmente en entornos universitarios, específicamente en la Universidad Técnica Nacional y en la Universidad Nacional. Los resultados de la aplicación del aula invertida en dichos espacios evidencian aspectos favorables, tanto para docentes como para estudiantes, debido a que el ambiente de la clase presencial se muestra más participativo y reflexivo, lo cual enriquece el proceso de aprendizaje (Arce, 2019) y, además, mejora el contenido académico estudiantil (Cordero y Núñez, 2017).

Las principales limitaciones encontradas se dirigen a la falta de acceso a recursos tecnológicos (Arce, 2019), además de la baja competencia para el uso de la tecnología con la que cuenta la población estudiantil y el cuerpo docente; también es una limitación el tiempo que se invierte en la auto instrucción, la aplicación de una cultura de autoaprendizaje y el desarrollo de las habilidades organizativas por parte de quienes aprenden (Cordero y Núñez, 2017).

\section{Marco teórico-CONCEPTUAL}

\subsection{Modelo pedagógico, modelos pedagógicos tradicionales y alternativos}

De acuerdo con Ortiz (2005), citado por Cantillo (2012), un modelo pedagógico es un "instrumento de la investigación de carácter teórico creado para reproducir idealmente el proceso enseñanza y aprendizaje (...) sirven para entender, orientar y dirigir la educación” (p.160). Según Vélez (2013), se caracterizan por el método expositivo de manera verbal para la transmisión del conocimiento, a lo cual se le conoce como magistralidad. Asimismo, explica que la evaluación suele ser sumativa, por lo cual el producto tiene mucho más valor que el proceso de aprendizaje en sí. Según el autor, debido a ello, estos modelos sostienen un clima desfavorable para el fomento del aprendizaje, pues se superpone la memorización de los contenidos, lo que otorga a las personas discentes un rol pasivo en el proceso educativo, aspecto que limita la creatividad y espontaneidad.

Además, se caracterizan por el uso de normas de clase rígidas (relación docente-estudiante vertical), que puede generar sentimientos de miedo y ansiedad, que llevan a las personas estudiantes a involucrarse poco en el proceso de aprendizaje y a cohibirse por miedo a recibir un castigo o una ridiculización (Franco,1997 citado en Vélez, 2013). Todas estas condiciones suelen afectar la autoestima y seguridad del alumnado, lo que lleva a inhibir los procesos cognitivos que motivan la apropiación del conocimiento, tales como la atención, concentración y memoria (Vélez, 2013).

Por su parte, las pedagogías alternativas emergentes durante el siglo XX (Gravié, 2018), se encuentran dentro del plano de la innovación, que pretende dar un giro a cómo se desarrollaba la educación tradicional del momento, es decir, un accionar pedagógico diferente al autoritarismo, la memorización de contenidos, y a un proceso centrado en la competencia y el individualismo. Además, según Pérez et al., (2016), las pedagogías alternativas,

esperan responder, incluso, a los estilos de aprendizaje de las personas, indistintamente de la edad, de las características cognitivas o del contexto socio cultural en el que se desarrollan. Estas rompen con el estilo convencional... pretenden producir cambios en el procedimiento del acto didáctico, para alcanzar una educación de recreación y más humanista; centrada en el ser, induciendo una modificación en la forma de alcanzar el aprendizaje; y con ello, los saberes y conocimientos (p. 238). 
Aunado a esto, Pérez et al., (2016) también menciona que en las pedagogías alternativas la interacción entre el estudiantado es uno de los principios centrales más importantes, pues se busca acentuar la máxima comunicación en el intercambio de conocimientos a través de novedosas prácticas escolares transformadoras que rechacen la idea de neutralidad, dependencia cognitiva, homogeneidad y pasividad, al promover procesos que se nutren de la heterogeneidad del estudiantado por medio de su participación activa.

\subsection{El Aula Invertida: Un modelo pedagógico alternativo}

Las investigaciones desarrolladas por diversas personas autoras conciben al aula invertida como un modelo pedagógico en donde, según Santiago y Bergman (2018),

El contenido básico es estudiado en casa con el material aportado por el profesor y el aula se convierte en un espacio de aprendizaje dinámico e interactivo, donde el docente guía a los alumnos mientras estos aplican lo que aprenden y se involucran con el objeto de estudio de manera creativa (p. 24).

Según estos mismos autores, dicha forma de generar aprendizajes combina dos elementos particulares, el primero es el espacio individual, es decir, asignaciones que son llevadas a cabo por las personas estudiantes en sus hogares y sin la ayuda del personal docente; y el espacio grupal, que ocurre en el aula, donde el profesorado funge como guía.

Por lo tanto, esta metodología busca invertir los roles tradicionales de la educación, pues en ella se brinda una estructura y dinámica diferente, donde además se procura que los procesos de aprendizaje sean más democráticos e inclusivos; democráticos debido a que el estudiantado puede tener acceso al material que se va a trabajar en la clase presencial e incluso promover formas o dinámicas para abordarlo; e inclusivo porque permite a la persona docente dar atención especial al estudiantado con necesidades educativas especiales y asignar ejercicios diferentes a la virtualidad (Arce, 2019).

Asimismo, se busca que el estudiantado asuma un rol más activo dentro de su proceso educativo (Berenguer, 2016 citado en Aguilera-Ruiz et al., 2017); que se involucre más y que se apropie de su aprendizaje (Santiago y Bergman, 2018), pues esta práctica permite la posibilidad de hacer búsquedas en internet y realizar ejercicios o indagaciones que les permitirán ir construyendo su propio conocimiento (Arce, 2019); también se señala que el aula invertida no implica menos trabajo para la persona docente, sino, más bien, requiere una clara planificación y mucho compromiso.

Es importante recalcar que en este modelo de trabajo las clases presenciales no son eliminadas, pues, aunque sí se trabaja virtualmente o en casa, lo que se busca es optimizar el tiempo de aula con experiencias de aprendizaje que fortalezcan lo estudiado, con ayuda de la persona docente y los demás compañeros y compañeras. Por último, se rescata que este modelo es perfectamente aplicable en todas las áreas curriculares, es decir, en educación primaria, secundaria, superior e, incluso, en educación para adultos (Blasco, Lorenzo y Sarsa, 2016, citado en Aguilera-Ruiz et al., 2017).

\subsubsection{Fases para la implementación del aula invertida}

Quesada (2018, citado en Arce, 2019) afirma que para la aplicación del aula invertida se requiere el cumplimiento de tres fases o procesos:

a) Pre-clase: tiene como objetivo la transferencia de los contenidos y se refiere a todos los trabajos que se realizan antes de la clase. La persona docente debe planificar todos los materiales: videos (YouTube), lecturas y tareas en físico o indagaciones pre-clase, uso de blogs y wikis, entre otros, para abordar el contenido (se toma en cuenta la accesibilidad que tenga el alumnado). El estudiantado debe comprometerse a realizar las actividades individuales o incluso grupales, previas a la clase, para transferir el conocimiento que se va 
a profundizar y participar en las actividades a desarrollar (Quesada, 2018 citado en Arce, 2019; Gimeno y Rodríguez, 2011; Adell, 2007; Ramírez-Ochoa, 2016).

b) Clase: suele ser presencial, busca promover una dinámica activa en el abordaje de los contenidos. El personal docente parte del hecho de que las personas discentes llegan preparadas a la lección, pues han realizado por sí mismas las actividades que fueron asignadas en la pre-clase. Es indispensable que la persona docente promueva la participación del alumnado para que el aprendizaje sea significativo (Quesada, 2018 citado en Arce, 2019).

Durante la clase, por lo general, se utilizan recursos didácticos que fomentan la participación activa del estudiantado, estrategias de aprendizaje entre iguales o pares, a partir del criterio "el mejor maestro de un niño es otro niño" (Gravié, 2018, p.74), con especial aplicación de la siguiente metodología:

- El aprendizaje colaborativo y cooperativo. Metodología que busca fomentar la participación grupal y social dentro del aula. Como menciona Ariño (2017), en el aprendizaje cooperativo el control y dirección del aprendizaje es guiado por la persona docente que diseña los objetivos; mientras que, en el aprendizaje colaborativo, las personas estudiantes tienen mayor libertad para aprender a su modo. Este tipo de abordaje dentro de la clase según Andreu (2016) genera que "el aprendizaje se vuelva más efectivo cuando los estudiantes trabajan en equipo" (p. 1045), porque les permite expresar sus opiniones y pensamientos, con el fin de resolver un problema.

Algunas de las técnicas utilizadas en esta metodología son: Puzzle de Aronson, Juego-Concurso de De Vries, grupo de investigación (García et al., 2019), tutorización, el folio giratorio, la petición en rueda (roundrobin), la entrevista en tres pasos, rincones, parejas a ciegas (Match Mine), respuesta juntos (Numbered Heads Together), el círculo dentro-fuera (Inside-Outside Circle), revisión por parejas (Pairs Check), piensa coopera comparte (Think-Pair-Share), mesa redonda (Roundtable) y la pizarra compartida (Blackboard Share) (Trujillo y Ariza, 2006; López y Acuña, 2011).

c) Post-clase: es una evaluación que permite comprobar si se dio un aprendizaje. Esta evaluación, en muchos de los casos, es formativa, aunque podría tener carácter sumativo; para realizarla se pueden utilizar diferentes medios tecnológicos o incluso medios tradicionales al final de la clase. Siempre busca que el estudiantado participe en la evaluación y logre corroborar su propio aprendizaje (Quesada, 2018 citado en Arce, 2019).

\section{Metodología}

\subsection{Método}

En el presente artículo se desarrolla un estudio de caso, el cual que se ve inserto dentro del enfoque cualitativo de investigación. Con este se buscó indagar las experiencias de las personas participantes sobre la utilización del Aula Invertida como estrategia metodológica en sus clases, a nivel de secundaria en el Centro Educativo CEDES Don Bosco. Dada la escasa investigación acerca de la temática a nivel nacional, este estudio se presenta como una aproximación investigativa con un alcance exploratorio.

\subsection{Participantes}

Los criterios de inclusión: 1) que fueran personas docentes activas de CEDES Don Bosco o personas funcionarias de la institución, y 2) que hayan tenido experiencia de haber aplicado la metodología dentro de esta institución educativa. Las personas participantes elegidas son dos docentes mujeres que imparten clases de Español (participante 1) y Dibujo Técnico (participante 2), y un psicopedagogo que actualmente labora en el Equipo de Innovación Pedagógica (EIP) de la institución (participante 3). 
Estas personas se eligieron debido a la completa trayectoria que han tenido en todo el proceso de capacitación e implementación del modelo pedagógico en la institución estudiada. El método de elección de las personas participantes fue por conveniencia, de acuerdo con el criterio de las investigadoras; además, por la existencia de un nexo con una persona participante, quien se eligió por su destacada labor en el tema estudiado en esta institución específica; sugiere, a dos participantes, que fueron contactadas por correo electrónico, y que aceptaron participar bajo condiciones de anonimato.

\subsection{Procedimiento y análisis de los datos}

En un primer momento, se seleccionó a tres personas funcionarias del Centro Educativo CEDES Don Bosco que han aplicado el aula invertida en sus clases. Se les pidió colaboración en el proyecto y se les envió por correo electrónico el consentimiento informado. Seguido a la firma del consentimiento, las entrevistas fueron realizadas por las investigadoras mediante la plataforma Zoom, con cada participante por separado; se dedicó un tiempo aproximado de dos horas a cada entrevista.

Seguidamente, se hizo la transcripción de dichas entrevistas para analizar su contenido y así conjugar categorías de información que lograran sistematizar los principales resultados obtenidos. En el análisis de datos, se realizó la identificación primaria de subcategorías temáticas que permitieran la codificación de la información, para después simplificar la información por medio de categorías principales que sistematizaron las temáticas detectadas; se respetó la cantidad de datos proporcionados.

Además, se realizó una triangulación de métodos y técnicas que, según Gurdián (2007), permite utilizar el contraste de información, en este caso con el uso de entrevistas realizadas a diferentes personas expertas, que permite hacer una indagación inicial del tema de interés por medio de categorías y subcategorías de análisis.

\subsection{Guía de entrevista semiestructurada}

La guía de entrevista fue confeccionada a partir de los principales puntos teóricos develados en la bibliografía revisada sobre el aula invertida en espacios educativos. De este modo, las unidades de análisis empleadas para su confección son: conceptualización del aula invertida como modelo pedagógico, trayectoria en la implementación del aula invertida desde la experiencia de las personas entrevistadas, estrategias efectivas para los diferentes momentos del aula invertida, reacciones y actitudes presentadas por el estudiantado, aptitudes requeridas en las personas docentes, poblaciones y contextos de aplicación, y recomendaciones.

\subsection{Categorías y subcategorías de análisis}

A nivel general, se determinaron siete categorías principales. Con el uso de un cuadro se especifican las categorías-subcategorías y la interpretación semiótica de las tres entrevistas realizadas, como se muestra en la Tabla 1: 
TABLA 1.

Categorías y subcategorías de análisis

\begin{tabular}{|c|c|}
\hline Categoría & Subcategoría \\
\hline $\begin{array}{l}\text { Conceptualización del aula invertida } \\
\text { Importar lista. }\end{array}$ & $\begin{array}{l}\text { Uso empírico, definición, objetivo del } \\
\text { aula invertida. }\end{array}$ \\
\hline Implementación Importar lista & $\begin{array}{l}\text { Experiencia, utilización, conocimiento, } \\
\text { capacitaciones, proceso, cambio } \\
\text { metodológico, motivación, población, } \\
\text { papel institucional (enlace), papel del } \\
\text { sistema educativo. }\end{array}$ \\
\hline $\begin{array}{l}\text { Aplicación del aula invertida Importar } \\
\text { lista }\end{array}$ & $\begin{array}{l}\text { Metodologias, estrategias didácticas, } \\
\text { factores, espacio de trabajo } \\
\text { (antes-durante-después), aspectos } \\
\text { generales, evaluación, comunicación, y } \\
\text { apoyo del profesorado. }\end{array}$ \\
\hline $\begin{array}{l}\text { Beneficios y Aportes del aula invertida } \\
\text { Importar lista }\end{array}$ & $\begin{array}{l}\text { Beneficios al estudiantado, desarrollo de } \\
\text { habilidades en el alumnado, adquisición } \\
\text { de disciplina, adquisición de conciencia, } \\
\text { aprender a aprender, beneficios al } \\
\text { equipo docente, actualización } \\
\text { profesional, conocimiento de otras } \\
\text { estrategias, tiempo de calidad. }\end{array}$ \\
\hline Rol de la persona docente Importar lista & $\begin{array}{l}\text { Compromiso, planificación, } \\
\text { acompañamiento, motivación. }\end{array}$ \\
\hline Actitudes del estudiantado Importar lista & $\begin{array}{l}\text { Actitudes de adaptación y actitudes } \\
\text { requeridas. }\end{array}$ \\
\hline Retos Importar lista & $\begin{array}{l}\text { Retos, capacitación del profesorado, } \\
\text { apertura, creatividad, diseño de } \\
\text { asignaciones, población estudiantil, } \\
\text { percepción del estudiantado, } \\
\text { incertidumbre, adaptación del Aula } \\
\text { invertida, recursos, virtualidad, } \\
\text { evaluación, conocimiento de otras } \\
\text { metodologías, planificación, estructura, e } \\
\text { instrucción del estudiantado. }\end{array}$ \\
\hline
\end{tabular}

Fuente: Elaboración propia.

\section{RESUltados y ANÁLISIS DE LOS DATOS DE ENTREVISTA POR CATEGORÍAS PRINCIPALES}

\subsection{Conceptualización y qué busca el aula invertida}

Uno de los hallazgos se refiere al uso empírico de la metodología de aula invertida, ya que su implementación a veces se realiza "sin saber que está aplicando precisamente esa técnica. De pronto empezamos a hacer cosas donde queremos que los estudiantes participen más y no tener nosotros tanto el protagonismo sino ellos" (participante 1, comunicación personal, 23 de junio de 2020). Esto podría verse derivado del deseo de las personas docentes por construir un tipo de educación que sobrepase los límites de la educación tradicional. Asimismo, es importante resaltar que, a pesar de este inicio empírico en el uso de metodologías activas, se llevaron a cabo una serie de procesos de capacitación que permitieron tener una conceptualización más clara y fundamentada en torno al aula invertida, definida como: 
Darle la vuelta a la clase, hay varias literaturas que lo manejan como el aula patas para arriba... es dejar al estudiante investigar, se le deja una asignación... muy específica... y a partir de eso se empieza a trabajar en el aula presencial, o el aula sincrónica, y se aprovecha más el tiempo (participante 3, comunicación personal, 23 de junio de 2020).

Lo anterior coincide con lo encontrado en la teoría, ya que dentro de las investigaciones consultadas se plantea cómo esta metodología busca invertir los roles tradicionales de la educación mediante la creación de procesos de aprendizaje más democráticos e inclusivos, con el fin de optimizar el tiempo de las clases presenciales, y generar experiencias de aprendizaje que fortalezcan lo aprendido en casa (Arce, 2019).

Además, se reconoce que el aula invertida requiere de mucha planificación, ya que es "más estructurada...el aula invertida tiene un proceso, que se tiene que seguir y se tiene que conseguir" (participante 1, comunicación personal, 23 de junio de 2020). Así, se refuerza la necesidad de llevar una clara planificación y mucho compromiso (Arce, 2019), pues dicho proceso tiene un orden lógico y su correcta implementación permite el logro de aprendizajes significativos.

El objetivo que se persigue es que "el estudiante sea partícipe y dueño de todo el proceso del aprendizaje y que el profesor sea un facilitador” (participante 3, comunicación personal, 23 de junio de 2020). En síntesis, se busca un cambio de roles, que le devuelva al estudiantado su papel protagónico, donde pase de ser mero receptor de conocimientos a un constructor activo de aprendizajes.

\subsection{Proceso de implementación del aula invertida en CEDES Don Bosco}

Se mencionan diversas capacitaciones sobre el aula invertida y metodologías activas, como las recibidas por Progrentis y Mentora, a partir de una experiencia personal de una persona participante en el proceso de maestría, donde se implementó. Por lo tanto, se conversó que tuvieron sus primeros acercamientos hace unos 3 o 4 años aproximadamente.

Los elementos que los llevaron a elegir este tipo de metodologías son la anuencia al cambio, implementaciones nuevas alternativas y el evitar metodologías tradicionales, esto con el objetivo de hacer este proceso más dinámico, generar compromiso en el estudiantado, fomentar su participación activa y pensamiento crítico (participante 2, comunicación personal, 23 de junio de 2020).

En lo que respecta al proceso de implementación del aula invertida en CEDES Don Bosco, se menciona que fue un proceso que se dio a partir del establecimiento del Equipo de Innovación Pedagógica (EIP), y pasa por un enlace con las coordinaciones escolares; ya que, al haber apoyo institucional, se facilitó esa transición hacia la aplicación de metodologías activas e innovadoras.

Asimismo, "la manera de ir cambiando al aula invertida ha sido... un desafío, a veces un reto, porque también los estudiantes no están acostumbrados ni los papás” a su aplicación (participante 2, comunicación personal, 23 de junio de 2020). Ha resultado necesario dar a conocer estas metodologías, sus beneficios, e inculcar en las personas estudiantes una cultura de responsabilidad, para que puedan asumir las asignaciones que se les dejan para la casa.

El aula invertida se constituye como una metodología que puede ser perfectamente aplicable en los diversos escenarios educativos como "primaria, secundaria, e incluso preescolar" (participante 3, comunicación personal, 23 de junio de 2020). Para lograr la aplicación de esta metodología en los diversos niveles, es necesario que esta se dé en forma "estructurada y organizada" (participante 2, comunicación personal, 23 de junio de 2020).

En CEDES Don Bosco, la innovación pedagógica ha sido promovida por las personas dirigentes, incluso al punto de plantearse que "la institución ha sido muy abierta y por eso ha creado el Equipo de Innovación Pedagógica, justamente para que este tipo de metodologías se puedan ir aplicando y darlas a conocer" (participante 2, comunicación personal, 23 de junio de 2020). Es decir, no basta con el deseo de las personas docentes por mejorar la educación, sino que también depende de las acciones de los centros 
educativos, pues los últimos pueden facilitar o no la implementación de nuevas metodologías, que requieren además del interés, motivación y flexibilidad por parte de todas las personas involucradas.

El sistema educativo costarricense ha promovido la implementación de nuevas metodologías desde el año 2015, aproximadamente, al plantear la necesidad de hacer cambios en la forma en cómo se ha venido educando a la población (participante 2, comunicación personal, 23 de junio de 2020).

\subsection{Aplicación del aula invertida en CEDES Don Bosco}

En esta subcategoría resaltan tres elementos: el equipo docente considera los recursos y las diferencias grupales que tiene el estudiantado para formular sus actividades, dado que la adecuación del aula invertida al entorno responde a las diferencias socioeconómicas y la inclusión de toda persona estudiante por medio de estrategias que permitan desarrollar el aula invertida (participante 2; participante 1 comunicación personal, 23 de junio de 2020).

También destacan que es indispensable el proceso de instrucción sobre la actividad que se hará en casa, sobre todo si esta requiere medios virtuales y si no ha sido utilizada antes por las personas estudiantes. Por ello, se debe "tratar de enviarles la instrucción más clara pero no darles de más, porque la idea es que ellos investiguen" (participante 3, comunicación personal, 23 de junio de 2020).

Por último, se refiere a la intermitencia con la que se debe aplicar el aula invertida, pues se trata de ejecutar al menos tres veces durante el trimestre, en coordinación con todo el equipo docente. Esto debido a la recomendación de que "no se hiciera todas las semanas, porque sabíamos que muchos docentes iban a estar también utilizando este tipo de modalidad" (participante 3, comunicación personal, 23 de junio de 2020), así se podría evitar la sobrecarga estudiantil.

El proceso de aula invertida que se aplica en CEDES consta de tres pasos principales: el antes, el durante y el después. El antes se refiere al primer contacto del estudiantado con el tema, esto mediante el trabajo en casa y por medio de un insumo y una asignación pequeña. Las estrategias didácticas pueden ser tanto virtuales (uso de podcasts, videos, lapbooks, diseño de recursos audiovisuales varios, portafolios virtuales, investigación, uso de plataformas como Classroom y Padlet) como no virtuales (lectura de cuentos, estudios de casos, asignación de cuestionarios breves, mapas mentales, investigación, estudio del entorno, bitácoras).

El durante se refiere al abordaje de la temática en clase, donde la persona docente facilita actividades que giran en torno a dos estrategias didácticas esenciales: el aprendizaje colaborativo (foros, conversatorios, mesas redondas) y el aprendizaje basado en proyectos (creación de maquetas, estructuración de programas de radio, diseño de campañas publicitarias, etc.), donde se construye el proceso de enseñanza y aprendizaje mediante la socialización y el trabajo en conjunto con sus compañeros y compañeras (participante 3; participante 2; participante 1, comunicación personal, 23 de junio de 2020).

El después se dirige a la socialización de aprendizajes (exposiciones en la feria de arte y tecnología, diseñados en procesos de aula invertida). Además, permite la expresión, asociación personal y emocional sobre las diferentes vivencias con la materia (participante 1, comunicación personal, 23 de junio de 2020).

Finalmente, las personas entrevistadas enfatizaron que la aplicación del aula invertida en CEDES Don Bosco está inmersa dentro de un foco de evaluación formativa, que trata de contemplar el avance del estudiantado a lo largo de los tres pasos: "la evaluación es continua,... importa desde el inicio, la mitad y el final del proceso" (participante 3, comunicación personal, 23 de junio de 2020). Ante la complejidad que puede involucrar la evaluación del paso antes, se destacó el uso de registros como bitácoras, fotografías, portafolios o la dedicación de un espacio para comentar la actividad y valorar el espacio de trabajo realizado en casa. 


\subsection{Beneficios del aula invertida}

Los beneficios se sistematizan en dos subcategorías: beneficios hacia el estudiantado y beneficios hacia el equipo docente. En el primero se aportan estrategias de vida que van más allá de la comprensión teórica de la materia, tales como la adquisición de autodisciplina, aprender a aprender y autoconsciencia en el desarrollo de su propio proceso de aprendizaje, un mayor conocimiento tecnológico y un sentido de aplicabilidad de los conocimientos adquiridos a su propia vida. Asimismo, el estudiantado que no estuvo involucrado en los procesos de aprendizaje particulares de cada subgrupo también se ve beneficiado, esto gracias a la socialización que se realiza en el paso después, cuyo objetivo es que "esa información y ese conocimiento se comparta con los demás” (participante 3, comunicación personal, 23 de junio de 2020).

Con respecto al profesorado, las personas entrevistadas mencionan que permite la actualización de conocimientos en diferentes estrategias didácticas, gracias a la capacitación y a la cualidad investigativa que la misma metodología promueve. En línea con esto, dicha implementación ha generado un cambio de pensamiento más orientado hacia la innovación pedagógica (participante 1, comunicación personal, 23 de junio de 2020).

Para el caso particular de CEDES Don Bosco, se enfatiza que el aula invertida "es una metodología que ha llegado para facilitar el trabajo del docente, que sabemos que es una profesión bastante llena de trabajo, llena de asignaciones y que más bien esto viene a facilitar el proceso de enseñanza aprendizaje" (participante 3, comunicación personal, 23 de junio de 2020), pues la metodología brinda mayor tiempo de calidad en clase, debido a que el estudiantado llega con una preparación previa a la temática que se aborda en el aula, y es quien se encarga activamente de construir sus conocimientos mediante la facilitación brindada por el equipo docente.

\subsection{Rol de la persona docente}

El rol docente, en el caso de la estrategia del aula invertida, es fundamental porque "se busca invertir los roles" (participante 2, comunicación personal, 23 de junio de 2020); eso no exime al profesorado de las funciones que desempeña. Se destacan cuatro aspectos que son básicos: el compromiso del personal docente, la planificación, el acompañamiento y la motivación.

Además, se señala que el equipo docente debe mostrar compromiso al no conformarse con hacer una clase tradicional o con hacer el mínimo esfuerzo, sino que se debe comprometer a reformular su práctica educativa, para así motivar más al estudiantado (participante 1, comunicación personal, 23 de junio de 2020). Se entiende que "el aprendizaje es por la sorpresa, por la motivación y si yo, como docente, no tengo esas primicias desde el inicio, no va a servir cuanta metodología haga" (participante 3, comunicación personal, 23 de junio de 2020). Esta motivación "está ligada a la vocación y a su gusto por hacer una clase diferente” (participante 2, comunicación personal, 23 de junio de 2020). La persona docente se preocupa por la búsqueda de material para hacer sus clases más dinámicas (participante 1, comunicación personal, 23 de junio de 2020).

Existe un compromiso por capacitarse, pues, en CEDES Don Bosco, las capacitaciones han jugado un papel muy importante en el personal docente, "cuando va a congresos, o busca capacitaciones extra, es porque está convencido de que quiere dar una educación de calidad" (participante 1, comunicación personal, 23 de junio de 2020). También, se menciona la importancia de la disciplina de la persona docente a la hora de planificar, al evitar a toda costa hacer improvisaciones; se debe investigar y probar antes de aplicar las estrategias.

Para la planificación se debe tomar en cuenta la interdisciplinariedad, el contexto, los recursos y las realidades de las personas estudiantes, pues innovar no implica solamente usar tecnología, sino también saber adaptar las estrategias a la realidad en la que se imparten las clases; esto implica tener innovación para 
adaptar las actividades para quienes no tienen acceso, es decir, tener cierto grado de flexibilidad. En el caso de que una persona estudiante no haya podido participar o hacer alguna asignación, la persona docente debe asignarle algún otro trabajo, con el fin de que se haga responsable (participante 1; participante 2; participante 3, comunicación personal, 23 de junio de 2020)

$\mathrm{Al}$ inicio, el alumnado puede poner muchas excusas para no realizar los trabajos en casa, y estas pueden estar relacionadas o no con el acceso a los recursos que el estudiantado tenga; por lo tanto, el profesorado debe de prepararse con diferentes opciones para que la clase no sea saboteada (participante 1, comunicación personal, 23 de junio de 2020). Otro aspecto señalado a la hora de planificar es tener claridad en los objetivos que se persiguen en la materia, “'qué es lo que yo quiero que aprendan mis estudiantes?” (participante 2, comunicación personal, 23 de junio de 2020), ¿cuál es su perfil de salida?

En el acompañamiento es importante que la persona docente se comunique con claridad, que se asegure de que las instrucciones son entendidas por todo el estudiantado; por tanto, se requiere conocer al alumnado, pues de esa manera se sabe cuáles estrategias son más oportunas para las diferentes personas, es decir, se debe conocer y tener buena comunicación con el estudiantado, por lo cual hay que ser muy ordenado y organizado (participante 2, comunicación personal, 23 de junio de 2020).

Con respecto al uso de la tecnología, la participante 1 y el participante 3 (comunicación personal, 23 de junio de 2020) mencionan que muchas veces las personas adultas asumen que el estudiantado sabe usar la tecnología por el sólo hecho de nacer en la era digital, pero en realidad resulta necesario capacitar a las personas usuarias en la herramienta que se va a utilizar.

El personal docente, en su labor de acompañamiento, debe enfocarse en el proceso y no en la nota, al respecto se indica lo siguiente: "¿Qué es lo que viene el aula invertida a cambiar? Que no nos interesan tanto las notas; lo que interesa es el proceso durante ese aprendizaje" (participante 2, comunicación personal, 23 de junio de 2020). Añadido a esto, "es el docente quien va a supervisar todo el proceso" (participante 3 , comunicación personal, 23 de junio de 2020), es decir, la persona docente no sólo califica trabajos finales y da notas, sino que se encarga de supervisar el proceso de aprendizaje del estudiantado.

Finalmente, se enfatiza que el equipo docente debe ser resiliente en tanto a la aplicación de diferentes estrategias didácticas en el aula invertida, pues es importante no desistir e intentarlo varias veces si es necesario (participante 1, comunicación personal, 23 de junio de 2020).

\subsection{Actitudes del Estudiantado}

Esta categoría se divide en dos subcategorías: actitudes de adaptación y actitudes requeridas para la implementación del aula invertida. En actitudes de adaptación se señala que existen dos tipos de personas estudiantes: el primer grupo estudiantil que señala la participante 1 son estudiantes que generalmente se comprometen, cumplen con sus asignaciones y participan activamente; sin embargo, el reto de la persona docente es con las actitudes de irresponsabilidad y pereza que presentan algunas personas estudiantes. Menciona que "al principio se sienten molestos... pero llega un momento en que ellos lo hacen" (participante 1, comunicación personal, 23 de junio de 2020); también hay estudiantes que "no les gusta leer... quieren más bien que todo se los diga, o que todo sea por vídeo" (participante 2, comunicación personal, 23 de junio de 2020).

Las actitudes mencionadas pueden ser comunes en el alumnado especialmente al inicio, pues "es típico del ser humano poco a poco, irse acoplando a esta nueva normalidad, a esta nueva metodología" (participante 3 , comunicación personal, 23 de junio de 2020), debido a esto el personal docente debe ser firme en buscar la manera de cambiar la cultura facilista del estudiantado y promover el desarrollo de las siguientes actitudes: 
- Responsabilidad y compromiso para hacer el trabajo; cumplimiento de objetivos del aula invertida; la iniciativa "que pueda seguir una guía y tenga comprensión de lectura, no solo hacer por hacer, sino comprender para qué sirve” (participante 3, participante 2, comunicación personal, 23 de junio de 2020).

- Una actitud activa requiere que "el estudiante no esté paralizado, sino que... tiene que ser partícipe, tiene que estar interesado" (participante 2, comunicación personal, 23 de junio de 2020) durante todo su proceso de aprendizaje.

\subsection{Retos}

Sobre los retos al utilizar el aula invertida, una de las preocupaciones que surgen es el

qué van a decir los padres de familia de lo que se está haciendo, principalmente porque se podría pensar de forma errónea que el aula invertida no funciona, o que se espera una clase normal, donde todos estén en silencio, o cuando a nivel institucional la jefatura no está convencida de que eso se pueda aplicar en su aula (participante 1, comunicación personal, 23 de junio de 2020).

El reto del personal docente es la aplicación de estrategias innovadoras, sin la preocupación de no saber cómo utilizarlas, por ansiedad o miedo a que vaya a salir mal (participante 3, comunicación personal, 23 de junio de 2020).

También es necesario buscar espacios de apertura, para eliminar actitudes negativas con respecto a estas innovaciones, como pensar que no sirven sin haberlo intentado; se debe fomentar la creatividad en la persona docente, para impulsar el compromiso y motivación del estudiantado a realizarlas (participante 2, comunicación personal, 23 de junio de 2020). Ante lo anterior, se expresó lo siguiente:

podemos tener un colegio técnico con todas las facilidades de infraestructura, internet de banda ancha, todos los insumos tecnológicos, que si yo como docente digo: me da miedo hacerlo, no quiero hacerlo, no lo voy a hacer... ahí está la limitante (participante 3, comunicación personal, 23 de junio de 2020).

La situación anterior podría ser observada por el estudiantado y generar en este percepciones de falta de acompañamiento que conlleven a la incertidumbre.

Para mitigar estas situaciones de la percepción estudiantil, un reto que se ha enfrentado en CEDES Don Bosco es la adaptación del Aula Invertida al contexto y necesidades del estudiantado. Se toma en cuenta que la realidad del aula es muy diferente a una realidad de texto teórico, puesto que a veces se debe adaptar el currículum al estudiantado, al considerar las dificultades en el acceso a medios, principalmente al uso de tecnologías, sin que la familia perciba esto como discriminatorio, ya que no todas las personas estudiantes cuentan con los mismos recursos, ni con el mismo perfil económico, por lo que el profesorado debe estar abierto a adaptar las actividades (participante 1, comunicación personal, 23 de junio de 2020).

Finalmente, el último reto que se evidencia en las entrevistas tiene que ver con la evaluación, pues esta podría tornarse complicada, principalmente con la situación actual de la pandemia; de ahí que resulta importante lograr entender que, aunque sea evaluación formativa y que incluso esta pueda resultar difícil al momento de empezar a crear las rúbricas (participante 2, S. Hernández, comunicación personal, 23 de junio de 2020), sí vale el esfuerzo.

\section{Conclusiones}

El aula invertida se constituye como una metodología activa que busca trascender las concepciones tradicionales acerca de la educación, así como generar un cambio de roles que permita el reconocimiento de la persona estudiante como protagonista de los escenarios educativos y en sus procesos de enseñanza y aprendizaje. 
Son muchos los beneficios que se desprenden de su uso, entre estos la posibilidad que brinda de maximizar el tiempo destinado a las clases presenciales. Estas metodologías, en lugar de llevar a la reducción de los contenidos curriculares, permiten un mejor aprovechamiento para generar aprendizajes significativos.

Tal como se observa en los resultados y análisis de este estudio, la aplicación del aula invertida en la institución CEDES Don Bosco ha permitido corroborar su efectividad, evidenciada en la literatura internacional. Además, entender a nivel general el concepto que se tiene sobre el modelo, el proceso de implementación en la institución, la forma de aplicación, los roles y actitudes de las personas docentesestudiantes, los beneficios y los diferentes retos enfrentados.

La aplicación de este modelo pedagógico requiere de personas docentes comprometidas, que dediquen tiempo a investigar, capacitarse y planificar, ser creativas, empáticas, comunicativas y con cierto grado de flexibilidad en las clases, de manera que activen la motivación del estudiantado, además, de la necesidad de adaptar las estrategias y herramientas utilizadas al contexto y particularidades del alumnado.

Se invita al personal docente a ser resiliente, ya que en la puesta en práctica del aula invertida se pueden dar dificultades (existir resistencia o actitudes de inconformidad por parte del estudiantado), pero a la luz de la teoría se plantea que es común en cualquier proceso de adaptación. Por esto, se requiere de perseverancia y sorpresa en las actividades innovadoras, para que con el tiempo se logre desarrollar un mayor compromiso en las personas discentes.

Por último, cabe mencionar que los objetivos establecidos en esta investigación fueron alcanzados en su totalidad, en vista de que se logró obtener aprendizajes vinculados a qué es el aula invertida, cuáles son sus características, e incluso se logró obtener de primera mano información acerca de cómo se ha implementado esta metodología dentro de una institución educativa costarricense. Además, se pudo apreciar las dificultades y ventajas que implica la puesta en práctica de esta, y el cómo puede ser utilizada dentro de cualquier tipo de contexto educativo, claro está que se toman en consideración las particularidades y posibilidades de la institución educativa, así como del estudiantado y del cuerpo docente.

\section{Referencias Bibliográficas}

Adell, J. (2007). Wikis en educación. En: J. Cabero, y J. Barroso (Eds.), Posibilidades de la teleformación en el espacio europeo de educación superior (pp. 323-333). Editorial Octaedro. https://www.researchgate.net/publication/21 6393184_Wikis_en_educacion

Aguayo, M., Bravo, M., Nocetti, A., Concha, L. y Aburto, R. (2019). Perspectiva estudiantil del modelo pedagógico flipped classroom o aula invertida en el aprendizaje del inglés como lengua extranjera. Revista Educación, 43(1), 2215-2644. https://doi.org/10.15517/revedu.v43i1.31529

Aguilera-Ruiz, C., Manzano-León, A., Martínez-Moreno, I., Lozano-Segura, M. C. y Casiano, C. (2017). El modelo flipped classroom. International Journal of Developmental and Educational Psychology, 4(1), 261-266. https://w ww.redalyc.org/pdf/3498/349853537027.pdf

Andreu, M. A. (2016). Aprendizaje cooperativo o colaborativo: ¿Hay alguna diferencia en la percepción de los estudiantes universitarios? Revista Complutense de Educación, 27(3), 1041-1060. https://doi.org/10.5209/rev_ RCED.2016.v27.n3.47398

Arce, A. A. (2019). EMP Flipped Classroom o Aula invertida. Revista Académica Arjé, 2(1), 27-32. http://revistas.u tn.ac.cr/index.php/arje/article/view/181/130

Ariño, M. L. (2017). Aprendizaje colaborativo y cooperativo. Universidad Marcelino Champagnat. https://bit.ly/3uK jBY8

Barao, L. y Palau, R. F. (2016). Análisis de la implementación de flipped classroom en las asignaturas instrumentales de $4^{\circ}$ educación secundaria obligatoria. Revista Electrónica de Tecnologia Educativa, 55, 1-13. https://doi.org/1 0.21556/edutec.2016.55.733 
Barros, V. M. y Martínez, M. B. (2018). Aula invertida en la enseñanza de Álgebra en la educación superior. Espirales: Revista multidisciplinaria de investigación, 2(13), 12-23. https://www.revistaespirales.com/index.php/es/articl e/view/150/101

Cantillo, A. V. (2012). Modelos pedagógicos: medios, no fines de la educación. Cuadernos de Lingüistica Hispánica, 19, 157-168. https://www.redalyc.org/pdf/3222/322227527008.pdf

Cordero, D. y Núñez, V. (2017). La enseñanza invertida (EI) o flipped teaching (FT) como método de mediación pedagógica aplicado a un curso de inglés integrado para otras especialidades en la Universidad Nacional (UNA), Costa Rica. Revista Ensayos Pedagógicos, 12(1), 85-110. https://doi.org/10.15359/rep.12-1.5

Coufal, K. (2014). Flipped learning instructional model: Perceptions of video delivery to support engagement in eighth grade math. Lamar University - Beaumont. ProQuest Dissertations and Theses, 208. https://search-proquest-com.ezproxy.sibdi.ucr.ac.cr/dissertations-theses/flipped-learning-instruct ional-model-perceptions/docview/1612629687/se-2?accountid $=28692$

Domínguez, L. C., Sierra, D., Pepín, J. J., Moros. G. y Villaraga, A. (2017). Efecto del Aula Invertida Extendida a simulación clínica para la resucitación del paciente traumatizado: estudio piloto de las percepciones estudiantiles sobre el aprendizaje. Revista Colombiana de Anestesiologia, 45(2), 4-11. https://doi.org/10.1016/j.rca.2017.07 .011

Estado de la Educación Costarricense. (2017). Los Programas de Equidad en la Educación Costarricense. https://www .estadonacion.or.cr/educacion2017/assets/ee6-informe-completo.pdf

Fúneme, C. (2019). El aula invertida y la construcción de conocimiento en matemáticas. El caso de las aplicaciones de la derivada. Tecné, Episteme y Didaxis: TED, 45, 159-174. https://doi.org/10.17227/ted.num45-9840

Galindo, J. J. y Badilla, M. G. (2016). Innovación docente a través de la metodología flipped classroom: percepción de docentes y estudiantes de educación secundaria.Didasc@lia: Didáctica y Educación, 7(6),153-172. https://dia lnet.unirioja.es/servlet/articulo?codigo $=6672798$

García, R., Traver, J. A., Candela, I. (2019). Aprendizaje cooperativo. Fundamentos, características y técnicas. CCS. htt ps://edicionescalasancias.org/wp-content/uploads/2019/10/Cuaderno-11.pdf

Gimeno, A. M. y Rodríguez, J. M. H. (2011). El blog como herramienta didáctica en el espacio europeo de educación superior. Pixel-Bit. Revista de Medios y Educación, 38, 165-175. https://recyt.fecyt.es/index.php/pixel/article/ view/61425

Gonçalves, Z. T. y Quaresma, D. (2018). Metodologia Ativa: Sala de Aula Invertida e suas Práticas na Educação Básica. Revista Iberoamericana sobre Calidad, Eficacia y Cambio en Educación, 16(4), 63-78. https://doi.org/10.15366 /reice2018.16.4.004

González, J. N. (2001). Hacia una reforma educativa en la era digital. Revista Iberoamericana de Educación, 26, 77-96. https://doi.org/10.35362/rie260979

Gurdián, A. (2007). El paradigma cualitativo en la investigación socio-educativa [archivo PDF]. Printcenter. https://web.ua.es/en/ice/documentos/recursos/materiales/el-paradigma-cualitativo-en-la-investi gacion-socio-educativa.pdf

Gravié, R. F. (2018). Una alternativa a la educación tradicional: el aprendizaje cooperativo. Revista Panamericana de Pedagogía. Saberes y Quehaceres del Pedagogo, 3, 73-84. https://bit.ly/3w1Hc6Y

Lage, M., Platt, G. y Treglia, M. (2000). Inverting the Classroom: A Gateway to Creating an Inclusive Learning Environment. The Journal of Economic Education, 31(1),30-43. https://doi.org/10.1080/00220480009596759

López, G. y Acuña, S. (2011). Aprendizaje cooperativo en el aula. Inventio. La Génesis de la Cultura Universitaria en Morelos, 14(7), 28-37. http://inventio.uaem.mx/index.php/inventio/article/view/381

Martínez-Olvera, W., Esquivel-Gámez, I. y Martínez-Castillo, J. (2014). Aula invertida o modelo invertido de aprendizaje: Origen, sustento e implicaciones. En Los Modelos Tecno-Educativos, revolucionando el aprendizaje del siglo XXI, (pp. 137-154). DSAE-Universidad Veracruzana. https://bit.ly/3blwHDy

Mendaña, C., Poy, R., González, A., Arana, M. V. y López, E. (2017). ¿Influye el aula invertida en la motivación y el rendimiento académico de estudiantes universitarios? Infancia, Educación y Aprendizaje (IEYA), 3(2), 660-666. https://doi.org/10.22370/ieya.2017.3.2.798 
Moya, P. y Williams, C. (2016). Efecto del Aula Volteada en el rendimiento académico: Estudio comparativo basado en el resultado del rendimiento académico con metodología Aula Volteada y Clase Tradicional para la asignatura de Salud Pública. Revista Educación Ciencias Salud, 13(1), 15-20. https://dialnet.unirioja.es/servlet/articulo?c odigo $=6289261$

Pavanelo, E. y Lima, R. (2017). Sala de Aula Invertida: a análise de uma experiência na disciplina de Cálculo I. Bolema: Boletim de Educação Matemática, 31(58), 739-759. http://dx.doi.org/10.1590/1980-4415v31n58a1 1

Peña, B., Zabalza, I., Usón, S., Llera, E., Martínez, A. y Romeo, L. (2017). Experiencia piloto de aula invertida para mejorar el proceso de enseñanza-aprendizaje en la asignatura de Termodinámica Técnica. En: In-Red 2017. III Congreso Nacional de Innovación Educativa y de Docencia en Red. Editorial Universitat Politècnica de València. http://dx.doi.org/10.4995/INRED2017.2017.6868

Pérez, Á. A., Gelves, B. B. A., Colmenárez, M. A. F. C. y Ramírez, T. E. C. (2016). Una aproximación a las pedagogías alternativas. Educere, 20(66), 237-247. https://www.redalyc.org/pdf/356/35649692005.pdf

Ramírez-Ochoa, M. I. (2016). Posibilidades del uso educativo de YouTube. RA ximhai, 12(6), 537-546. https://ww w.redalyc.org/pdf/461/46148194036.pdf

Sabater, M. P., Curto, J. J., Rourera, A., Olivé, M. C., Costa, S., Castillo, S. y del Pino-Gutiérrez, A. (2017). Aula invertida: experiencia en el Grado de Enfermería. Revista d'Innovació Docent Universitària, 9, 115-123. https:/ $/$ dialnet.unirioja.es/servlet/articulo?codigo $=5855834$

Santiago, R. y Bergmann, J. (2018). Aprender al revés: Flipped learning 3.0 y metodologias activas en el aula. Editorial PAIDÓS Educación. https://www.researchgate.net/publication/327040344

Trujillo, F. y Ariza, M.A. (2006). Experiencias educativas en aprendizaje cooperativo. Grupo Editorial Universitario. h ttps://bit.ly/33FlRnw

Vélez, A. M. (2013). Emergencias de cambio: entre el modelo pedagógico tradicional y la necesidad de aprendizajes significativos. Praxis, 9(1), 73-82. https://dialnet.unirioja.es/servlet/articulo?codigo=5907268

\section{INFORMACIÓN ADICIONAL}

Cómo citar: Araya-Moya, S. M., Rodríguez Gutiérrez, A. L., Badilla Cárdenas, N. F. y Marchena Moreno, K. C. (2022). El aula invertida como recurso didáctico en el contexto costarricense: Estudio de caso sobre su implementación en una institución educativa de secundaria. Revista Educación, 46(1). Recuperado de http ://doi.org/10.15517/revedu.v46i1.44333 